\title{
A Parametric Extension of Mixed Time/Frequency Robust Identification
}

\author{
Pablo A. Parrilo*
}

\author{
Mario Sznaier $\ddagger$
}

\begin{abstract}
A parametric extension to the time/frequency robust identification framework is presented. The result can be applied to plants which have a clear parametric and non parametric separation, on which time and/or frequency experiments have been performed. The parametric portion of the model should be affine in the unknown parameters, which includes practical applications such as flexible structures. The consistency problem is cast as a constrained finite-dimensional convex optimization problem that can be formulated as a Linear Matrix Inequality. The proposed procedure provides a convergent, interpolatory identification algorithm.
\end{abstract}

\section{Introduction}

During the past few years a large research effort has been devoted to the problem of developing deterministic identification procedures that, starting from experimental data and an a priori class of models, generate a nominal model and bounds on the identification errors. These models and bounds can then be combined with standard robust control synthesis methods (such as $\mathcal{H}_{\infty}$, $\ell_{1}$ or $\mu$-synthesis) to obtain robust closed-loop systems. This problem is usually known as the Robust Identification problem (see [6] for a survey).

Recent papers proposed interpolatory algorithms that use frequency domain data ([4]) or time domain experiments $([3,11])$ to generate a nominal model together with an $\mathcal{H}_{\infty}$ bound on the identification error. Furthermore, a new robust identification framework that takes into account both time and frequency domain experiments, has been introduced in [9] and generalized in [8]. Thus, the problem where "good" frequency response fitting (small $\mathcal{H}_{\infty}$ error norm) leads to "poor" fitting in the time-domain is prevented.

All of the above results address non parametric identification of models with a worst case global bound. In many cases, part of the model has a clear parametric structure, therefore disregarding this information may lead to conservative results. This is usually the case of mechanical

*P. Parrilo was Becario de Iniciación U.B.A., and is now with the Dept. of Control \& Dynamical Systems, California Institute of Technology. Email: pabloøcds.caltech.edu.

$\dagger_{R}$. Sánchez Peña is with the Depto. de Electrónica, Facultad de Ingeniería, Universidad de Buenos Aires, and also with the $\mathrm{Na}$ tional Commission of Space Activities (CONAE), Argentina. Email: ricardocconae.gov. ar

$\ddagger_{M}$. Sznaier is with the Dept. Electrical Engineering, Pennsylvania State University. Email: msznaier@frodo.ee.psu.edu flexible structures, which have a well defined parametric model for the lower frequency modes and an unknown higher frequency behavior which naturally leads to a non parametric identification ([5]).

This new result extends the time/frequency identification procedure in $[9,8]$ to the case of parametric/non parametric model structures, based on a similar framework. The parametric portion of the model should be affine in the parameters. This includes cases of practical interest as flexible mechanical structures, as well as models which can be concisely described in terms of a set of Laguerre or Kautz functions or, more generally, any other basis of $\mathcal{H}_{2}$.

The paper is organized as follows. In Section II we introduce a robust identification framework using both time and frequency experiments and some background material, including a generalized Nevanlinna-Pick theory ([1]), that contains as special cases, the classical CarathéodoryFejér and Nevanlinna-Pick problems. Section III contains the main theoretical results. Here we show that the problems of establishing consistency of the experimental data and the a priori information and of determining a nominal model can be recast into a finite-dimensional Linear Matrix Inequality (LMI) optimization form. Most of the proofs have been left out for space considerations. They can be found in [7]. In Section IV we illustrate the advantages of these results with a simple example.

\section{Robust Identification Framework}

The class of systems considered are discrete time, causal, linear and stable ones. We denote them as $H(z)=H_{d}\left(\frac{1}{z}\right)$, with $z \in \mathbb{C}$, and $H_{d}(z)$ being the usual $z$-transform. Therefore, causal stable systems $H(z)$ will be analytic inside the unit circle, with time and frequency representations related by:

$$
H(z)=\sum_{k=0}^{\infty} h(k) z^{k}
$$

For simplicity we consider SISO models, although all results can be applied to MIMO systems, following [3].

The $a$ posteriori experimental data can be obtained from two different sources: frequency response experiments and time domain data. For the first one, we will consider the set of $N_{f}$ samples of the frequency response of the system measured with additive bounded noise $y_{k}^{f}=\hat{h}_{k}+\eta_{k}^{f}, k=0, \ldots, N_{f}-1$, collected for notational simplicity in a vector $\mathbf{y}^{f} \in \mathbb{C}^{N_{f}}$. Here, $\hat{\mathbf{h}}$ is a vector 
with its components equal to $H\left(e^{j \Omega_{k}}\right), \quad k=0, \ldots, N_{f}-1$. The additive measurement noise $\eta^{f}$ is assumed to belong to a certain noise set, which is described below.

The time domain data considered is the set of the first $N_{t}$ time response samples to a known but otherwise arbitrary input, also corrupted by additive noise: $y_{n}^{t}=$ $(U h)_{n}+\eta_{n}^{t}, n=0, \ldots, N_{t}-1$.

$$
U=\left[\begin{array}{cccc}
u(0) & 0 & \ldots & 0 \\
u(1) & u(0) & \ldots & 0 \\
\vdots & \ddots & \ddots & 0 \\
u\left(N_{t}-1\right) & \ldots & u(1) & u(0)
\end{array}\right]
$$

is the Toeplitz matrix corresponding to the input sequence. Again, for notational simplicity, these measurements will be collected in a vector $\mathbf{y}^{t} \in \mathbf{R}^{N_{t}}$.

We will define our a priori class of noise in terms of linear matrix inequalities (LMIs). Such description is fairly general. In the present case, the LMI regions are useful to model convex noise sets because they provide a simple and exact way to take into account multiple point frequency response experiments.

In the time domain case, the noise is real-valued, and therefore convex sets are in fact just intervals. However, adopting an LMI-defined set can prove advantageous, for example, if the independence assumption on noise samples turns out to be conservative. In this case, the noise set is no longer the Cartesian product of the individual noise intervals.

In view of the preceding remarks, we define our $a$ priori noise sets $\mathcal{N}_{f}, \mathcal{N}_{t}$ as the feasibility regions of a set of LMIs.

$$
\begin{aligned}
& \mathcal{N}_{f} \triangleq\left\{\eta^{f} \mid L^{f}\left(\eta^{f}\right)=L_{0}^{f}+\sum_{k=0}^{N_{f}-1}\left[L_{k}^{f} \eta_{k}^{f}+\left(L_{k}^{f}\right)^{*} \eta_{k}^{f}\right]>0\right\} \\
& \mathcal{N}_{t} \triangleq\left\{\eta^{t} \mid L^{t}\left(\eta^{t}\right)=L_{0}^{t}+\sum_{k=0}^{N_{t}-1} L_{k}^{t} \eta_{k}^{t}>0\right\}
\end{aligned}
$$

where $\mathcal{N}_{f} \in \mathbb{C}^{N_{f}}, \mathcal{N}_{t} \in \mathbf{R}^{N_{t}}, L_{i}^{t}$ are given real symmetric matrices, and $L_{0}^{f}, L_{i}^{f}$ are hermitian and real square, respectively. We assume these a priori noise classes $\mathcal{N}_{f}, \mathcal{N}_{t}$ to be bounded.

Note that the $\ell_{\infty}(\epsilon)$ noise sets usually considered ([4, 8]) are just special cases of the above.

To introduce the a priori system class, define the following set:

$\mathcal{H}_{\infty}(\rho, K) \triangleq\left\{H(z)\right.$ analytic in $\left.\mathcal{D}_{\rho}\left|\sup _{z \in \mathcal{D}_{\rho}}\right| H(z) \mid<K\right\}$

where $\mathcal{D}_{\rho}$ is the open disk $\{z,|z|<\rho\}$ and $\overline{\mathcal{D}}_{\rho}$ is its corresponding closure. Also define $\mathcal{H}_{\infty}$ as $\mathcal{H}_{\infty, 1}, \mathcal{D}$ as $\mathcal{D}_{1}$ and $\overline{\mathcal{B}} \mathcal{H}_{\infty} \triangleq\left\{F \in \mathcal{H}_{\infty},\|F\|_{\infty} \leq 1\right\}$.
The class of models usually considered $\mathcal{H}_{\infty}(\rho, K)$ corresponds to exponentially stable systems (finite or infinite dimensional). From a practical viewpoint, these systems have a stability margin of $(\rho-1)$, a peak response to complex exponential inputs of $K$, and satisfy the following time domain bound:

$$
|h(k)| \leq K \rho^{-k}
$$

The above classes cover the nonparametric part of the $\operatorname{model} G_{n p}(z)$.

We seek the worst case identification of the combined parametric and non parametric models using both the time and frequency experiments, i.e. $G_{p}(z)+$ $G_{n p}(z)$. To this end we define the sets of systems $\mathcal{S} \triangleq$ $\left\{\mathcal{H}_{\infty}(\rho, K), \rho>1, K<\infty\right\}$ for the non parametric part and $\mathcal{P}$ for the parametric component $G_{p}(z)$.

The latter is the class of affine parametric models defined as follows:

$\mathcal{P} \triangleq\left\{\mathbf{p}^{T} \mathbf{G}(z), \quad \mathbf{p} \in \mathbb{R}^{N_{p}}, p_{i} \in\left[a_{i}, b_{i}\right], \mathbf{G}(z)\right.$ satisfies (3) $\}$

where the components $g_{i}(z)$ of vector $\mathbf{G}(z)$ are known linearly independent functions, which satisfy the separation condition:

$$
\operatorname{span}\left\{g_{i}(z)\right\} \cap \mathcal{S}=\{0\}
$$

This condition guarantees a unique parametric/non parametric decomposition for each element $G_{p}(z)+G_{n p}(z)$ of the a priori set. If this decomposition is not unique, then there are multiple parameter choices for each model in the a priori set. Therefore the parametric and nonparametric components will not converge separately, although the full model might converge to the real plant.

The underlying motivation for the above "clear separation" condition between the parametric and nonparametric components is as follows. There is a clear advantage of having a parametric component when it cannot be tightly described with a nonparametric model. In that case, a global nonparametric description would be unnecessarily conservative ([5]). On the other hand, in the case where the parametric portion can be included in the nonparametric one, there is no special reason to describe it parametrically. For all the previous arguments, we consider the case where the parametric and nonparametric components are clearly separated. This type of model description includes many practical situations, e.g. flexible structures. In the latter case, the parametric/nonparametric separation is in terms of the system natural frequencies. The lower frequencies have a parametric description, while the higher frequencies can be modeled nonparametrically.

Therefore, the a priori information and the a posteriori experimental input data are:

$$
\begin{aligned}
\mathcal{T} & =\left\{H(z)=G_{p}(z)+G_{n p}(z) \mid G_{p} \in \mathcal{P}, G_{n p} \in \mathcal{S}\right\} \\
\mathcal{N}_{f} & =\left\{\eta^{f} \in \mathbb{C}^{N_{f}}, \quad L^{f}\left(\eta^{f}\right)>0\right\}
\end{aligned}
$$




$$
\begin{aligned}
\mathcal{N}_{t} & =\left\{\eta^{t} \in \mathbf{R}^{N_{t}}, \quad L^{t}\left(\eta^{t}\right)>0\right\} \\
\mathbf{y}^{f} & =\left\{\hat{\mathbf{h}}+\eta^{f} \in \mathbb{C}^{N_{f}}\right\} \\
\mathbf{y}^{t} & =\left\{U \mathbf{h}+\eta^{t} \in \mathbf{R}^{N_{t}}\right\}
\end{aligned}
$$

The above a priori and a posteriori information allows a less conservative identification than in the cases where only time or frequency experiments are provided or when no knowledge of the parametric part of the model is used. Another interpretation can be made in terms of the "smaller" size of the consistency set, due to the fact that there are more experimental data and structured a priori information.

\subsection{Generalized Interpolation Frame- work}

In this section we briefly present a generalized interpolation framework developed in [1]. This framework will be used in Section III to solve the consistency problem.

Theorem 1 There exists a transfer function $F(z) \in$ $\mathcal{B} \mathcal{H}_{\infty}\left(\overline{\mathcal{B}} \mathcal{H}_{\infty}\right)$ such that:

$$
\sum_{z_{\circ} \in \mathcal{D}} \operatorname{Res}_{z=z_{o}} F(z) C_{-}(z I-A)^{-1}=C_{+}
$$

if and only if the following discrete time Lyapunov equation has a unique positive (semi) definite solution.

$$
M=A^{*} M A+C_{-}^{*} C_{-}-C_{+}^{*} C_{+}
$$

where $A, C_{-}$and $C_{+}$are constant complex matrices of appropriate dimensions. If $M>0$ then the solution $F(z)$ is non-unique and the set of solutions can be parameterized in terms of a linear fractional transformation of $Q(z)$, an arbitrary element of $\overline{\mathcal{B}} \mathcal{H}_{\infty}$.

Note that the matrices $A$ and $C_{-}$provide the structure of the interpolation problem while $C_{+}$provides the interpolation values. It can be shown ([1]) that both the Nevanlinna-Pick and the Carathéodory-Fejér problems are special cases of this theorem, corresponding to an appropriate choice of the matrices $A$ and $C_{-}$,

\section{Main Results}

Nevanlinna-Pick based identification algorithms address the case where the experimental data available is purely frequency domain ([4]), while Carathéodory-Fejér based identification deals only with time domain data ([3]). In this section we exploit the generalized interpolation framework introduced in the previous section to solve Problem 1, obtaining a robust identification algorithm that combines both sources of data. To this effect, we divided Problem 1 into two subproblems: i) consistency and ii) identification. The first should determine the existence of a candidate model $H \in \mathcal{T}$ which may have produced both, the time and frequency domain experimental data. Clearly, this is a prerequisite to the second stage, the identification step, consisting of the computation of the nominal model itself and a bound on the identification error.

\subsection{Consistency}

We will see now that the problem of determining consistency of the a posteriori and a priori information reduces to establishing whether or not there exists a model $H \in \mathcal{T}$ that interpolates the frequency experimental data within the noise bounds:

$$
\hat{\mathbf{h}}=\mathbf{y}^{f}-\eta^{f}, \quad \eta^{f} \in \mathcal{N}_{f}
$$

and has an impulse response that satisfies the following constraints:

$$
U \mathbf{h}=\mathbf{y}^{t}-\eta^{t}, \quad \eta^{t} \in \mathcal{N}_{t}
$$

where the noiseless output $U \mathbf{h}$ is the convolution of the input vector $u^{T}=\left[\begin{array}{llll}u(0) & u(1) & \ldots & u\left(N_{t}-1\right)\end{array}\right]$ and the system $H(z)$.

The main result of this section shows that consistency can be established by solving a finite-dimensional convex optimization problem. In Theorems 2 and 3 we will show that this generalized problem can be recast in terms of an LMI optimization.

The next theorem provides necessary and sufficient conditions for the existence of a function $H \in \mathcal{H}_{\infty}(\rho, K)$ which interpolates fixed frequency domain experimental data while, at the same time, satisfies a time-domain constraint.

Theorem 2 Given $N_{f}$ frequency-domain data points, $H\left(z_{i}\right)=w_{i}, \quad i=0, \ldots, N_{f}-1,\left|z_{i}\right|<\rho$ and $N_{t}$ timedomain data points $h(k), k=0, \ldots, N_{t}-1$, there exists $H \in \mathcal{H}_{\infty}(\rho, K)$ that interpolates the frequency domain data and such that $H(z)=h(0)+h(1) z+h(2) z^{2}+\ldots+$ $h\left(N_{t}-1\right) z^{N_{t}-1}+\ldots$ if and only if

$M_{R}(\mathbf{w}, \mathbf{h})=\left[\begin{array}{cc}Q-\frac{1}{K^{2}} \mathcal{W}_{f}^{*} Q \mathcal{W}_{f} & M_{X} \\ M_{X}^{*} & R^{-2}-\frac{1}{K^{2}} \mathcal{F}_{t}^{*} R^{-2} \mathcal{F}_{t}\end{array}\right]>0$

where

$$
\begin{aligned}
M_{X} & =S_{0} R^{-2}-\frac{1}{K^{2}} \mathcal{W}_{f}^{*} S_{0} R^{-2} \mathcal{F}_{t} \\
R & =\operatorname{diag}\left[\begin{array}{ccccc}
1 & \rho & \rho^{2} & \ldots \rho^{N_{t}-1}
\end{array}\right] \\
Q & =\left[\frac{\rho^{2}}{\rho^{2}-\bar{z}_{i} z_{j}}\right]_{i j} \\
S_{0} & =\left[\begin{array}{ccccc}
1 & \bar{z}_{0} & \bar{z}_{0}^{2} & \ldots & \bar{z}_{0}^{N_{t}-1} \\
1 & \bar{z}_{1} & \bar{z}_{1}^{2} & \ldots & \bar{z}_{1}^{N_{t}-1} \\
\vdots & \vdots & \vdots & \vdots & \vdots \\
1 & \bar{z}_{N_{f}-1} & \bar{z}_{N_{f}-1}^{2} & \ldots & \bar{z}_{N_{f}-1}^{N_{t}-1}
\end{array}\right]
\end{aligned}
$$




$$
\begin{aligned}
\mathcal{W}_{f} & =\operatorname{diag}\left[\begin{array}{lll}
w_{0} & \ldots & w_{N_{f}-1}
\end{array}\right] \\
\mathcal{F}_{t} & =\left[\begin{array}{cccc}
h(0) & h(1) & \ldots & h\left(N_{t}-1\right) \\
0 & h(0) & \ldots & h\left(N_{t}-2\right) \\
\vdots & \vdots & \ddots & \vdots \\
0 & 0 & \ldots & h(0)
\end{array}\right]
\end{aligned}
$$

Combining the previous result (that considers only noiseless data points) with the assumed parametric structure, we obtain the following necessary and sufficient condition for consistency:

Lemma 1 Define

$$
P_{t}=\left[g_{j}(i-1)\right]_{i j} \quad, \quad i=1, \ldots, N_{t}, j=1, \ldots, N_{p}
$$

and

$$
P_{f}=\left[G_{j}\left(z_{i-1}\right)\right]_{i j} \quad, \quad i=1, \ldots, N_{f}, j=1, \ldots, N_{p}
$$

Then, the a priori and a posteriori information are consistent if and only if there exists three vectors:

$$
\mathbf{p}=\left[\begin{array}{c}
p_{1} \\
p_{2} \\
\ldots \\
p_{N_{p}}
\end{array}\right], \mathbf{w}=\left[\begin{array}{c}
w_{0} \\
w_{1} \\
\ldots \\
w_{N_{f}-1}
\end{array}\right], \mathbf{h}=\left[\begin{array}{c}
h_{0} \\
h_{1} \\
\ldots \\
h_{N_{t}-1}
\end{array}\right]
$$

such that

$$
\begin{aligned}
M_{R}(\mathbf{w}, \mathbf{h}) & >0 \\
\left(\mathbf{y}^{f}-P_{f} \mathbf{p}-\mathbf{w}\right) & \in \mathcal{N}_{f} \\
\left(\mathbf{y}^{t}-U P_{t} \mathbf{p}-U \mathbf{h}\right) & \in \mathcal{N}_{t}
\end{aligned}
$$

Note that the components of $\mathbf{w}$ and $\mathbf{h}$ are elements of the matrices $\mathcal{W}_{f}$ and $\mathcal{F}_{t}$ respectively, and $g_{i}(k)$ the impulse response of $G_{i}(z)$.

From Lemma 1 it follows that the consistency problem can be reduced to solving a feasibility problem in terms of the parameter vector $\mathbf{p}$ and the time and frequency domain vectors $\mathbf{h}, \mathbf{w}$. The next Theorem shows that this feasibility problem is a convex problem that can be recast in terms of LMIs and thus efficiently solved, using for instance interior-point methods [2].

Theorem 3 The parametric/nonparametric consistency problem with mixed time/frequency-domain data is equivalent to a LMI feasibility problem.

An interesting question that appears at this point is related with the particular choice of a feasible solution for the LMI equations. For example, one could minimize the "size" of the noise set, under to the consistency restrictions. Or, maximize the smallest eigenvalue of the generalized Pick matrix, over the feasibility set, to avoid the ill-conditioning of the singular case. Alternatively, a minimization over $K$ (a bound on the norm of the nonparametric component) could be performed. From a control perspective, this latter option seems specially useful.

\subsection{Identification}

Once consistency is established, the second step towards solving the problem stated in Section II consists of generating a nominal model in the consistency set $\mathcal{T}\left(\mathbf{y}^{f}, \mathbf{y}^{t}\right)$. The identification algorithm that we propose is based on the parameterization of all solutions of the generalized Nevanlinna-Pick interpolation problem [1] presented in Theorem 1. For simplicity we consider the case where the matrix $M_{R}$ is strictly positive definite and therefore the solution is non-unique. Details for the degenerate case where there exists a unique solution can be found in [1]. The algorithm can be summarized as follows

1.- Find feasible data vectors $\mathbf{p}, \mathbf{w}, \mathbf{h}$ for the consistency problem (18)-(20) by solving the LMI feasibility problem.

2.- Compute the generalized Pick matrix $M_{R}$ in (8) (which should be positive definite), corresponding to the vectors computed in step 1.-.

3.- Use Theorem 1 to compute a model from the consistency set $\mathcal{T}$. Recall that all the models in $\mathcal{S}$ (i.e. all the solutions to the generalized interpolation problem) can be parameterized as a Linear Fractional Transformation (LFT) of a free parameter $Q(z) \in \overline{\mathcal{B}} \mathcal{H}_{\infty}$. In particular, if the free parameter $Q(z)$ is chosen as a constant, then the nonparametric model order is less than or equal to $N_{f}+N_{t}$.

Remark 1 Note that $T(z)$ depends on the choice of vectors $\mathbf{w}, \mathbf{h}$. Thus, there are additional degrees of freedom available in the problem (choices of $\mathbf{w}, \mathbf{h}$ and $Q(z)$ ) that could be used to optimize additional performance criteria (e.g. model order).

\subsection{Identification Error}

In [7] an upper bound for the worst-case identification error for the present problem is derived. Since this bound is given in terms of the radius and diameter of information [6], they are valid for all interpolatory algorithms taking as inputs the available a priori and a posteriori information (see lower bound in [8]).

An important point that needs to be emphasized at this stage lies on the fact that the identification error bounds are dependent on the assumed a priori information. That is, there will be different error bounds according to what is considered to be a priori . For example, by considering a fixed parametric component it is possible to change the a priori assumptions without necessarily changing the identified model, and keeping consistency in the process. In this case, the problem setup is nearly the same as the nonparametric case, and therefore the error bounds can be computed, for example, using the results in [8]. On the other hand, if we keep the a priori information structure of the parametric-nonparametric approach, weaker bounds can be obtained ([7]). 


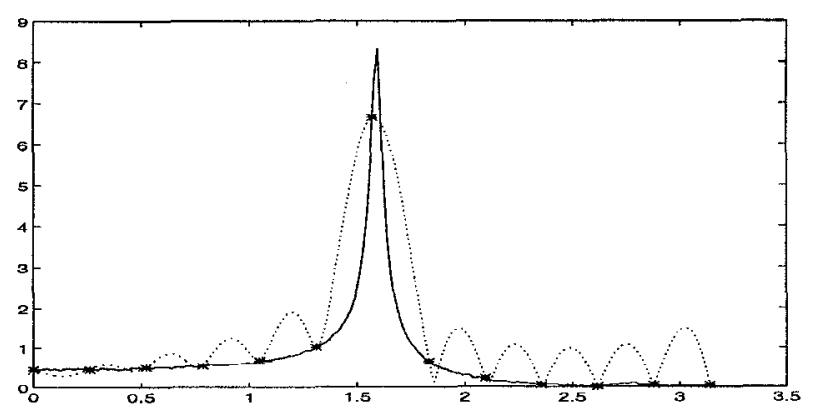

Figure 1: Nonparametric identification results. The full line is the physical system (with noise), and the dotted line the nonparametric identified model.

\section{Example}

In this section we present an application of the proposed identification procedure. The "experimental" data proceeds from the stable component (no rigid-body modes) of the Euler-Bernoulli model of a flexible bcam with viscous damping. For simplicity, we considered only frequency data, bilinearly-transformed from the original continuous-time samples.

We assumed a parametric component having the following (affine) structure:

$$
G(z)=\frac{p_{1} z+p_{2}}{z^{2}+0.04 z+1.05}
$$

where $p_{1}$ and $p_{2}$ are the uncertain parameters. The resonant poles of $G(z)$ were chosen according to the information available on the critical frequencies and damping factors of the plant. The optimization criterion chosen was to minimize the value of $K$, subject to the consistency restrictions.

In Figure 1 an identified model for the nonparametric identification procedure is shown. As noted above, in this approach the highly resonant poles force very small values of $\rho$ and/or large values of $K$, in order to satisfy the consistency constraints. This, in turn, causes larger error bounds, as well as less smooth interpolation functions. This issue is important in order to apply a subsequent model reduction stage.

Instead, when the approach developed in this note is employed, the complex dynamics of the parametric component can be "decoupled" from the nonparametric one. Figure 2 shows the identified parametric component, as well as the full model. Note that the parametric component is not able to explain, by itself, the whole dynamic behavior. Its objective is to model just one particular mode, rather than the entire transfer function. Note also that the identified nominal model is almost coincident with the experiment, providing a much better fit.

Acknowledgments- The work of the first two authors was supported by UBACyT projects IN018 and IN049, University of Buenos Aires. Mario Sznaier was supported by NSF under grant ECS-9625920.

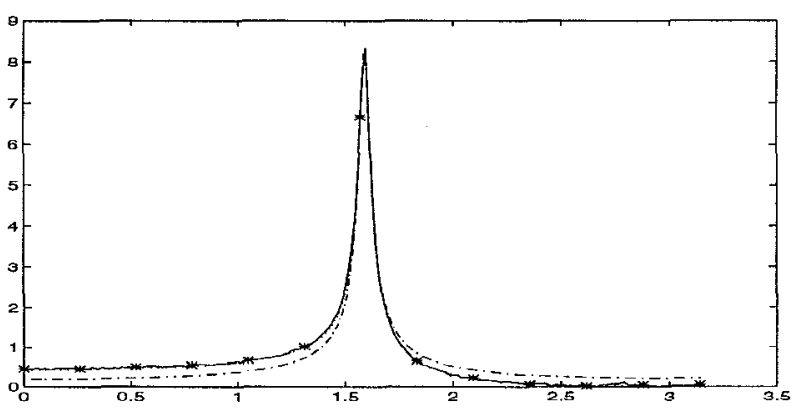

Figure 2: Mixed parametric/nonparametric identification results. The full line is the physical system (with noise), the dash-dotted line is the parametric model, and the dotted line is the full identified model.

\section{References}

[1] J. A. Ball, I. Gohberg, and L. Rodman, Interpolation of Rational Matrix Functions, Operator Theory: Advances and Applications, Vol. 45, Birkhäuser, 1990.

[2] S. Boyd, L. El Ghaoui, E. Feron, and V. Balakrishnan, Linear Matrix Inequalities in Systems and Control Theory. SIAM Studies in Applied Mathematics, Vol. 15, SIAM, Philadelphia, 1994.

[3] J. Chen, J. A. Farrell, C. N. Nett, and K. Zhou, " $\mathcal{H}_{\infty}$ Identification of Multivariable Systems by Tangential Interpolation Methods," IEEE Trans. Automat. Contr., Vol. 41, No. 12, 1996.

[4] J. Chen, C. N. Nett, and M. K. H. Fan, "Worst-Case System Identification in $\mathcal{H}_{\infty}$ : Validation of a Priori Information, Essentially Optimal Algorithms, and Error Bounds," IEEE Trans. Automat. Contr., Vol. 40, No. 7, 1995.

[5] J. Friedman and P. P. Khargonekar, "Application of Identification in $\mathcal{H}_{\infty}$ to Lightly Damped Systems: Two Case Studies," IEEE Transactions on Control Systems Technology, Vol. 3, No. 3, 1995.

[6] P. M. Mäkilä, J. R. Partington, T. K. Gustafsson, "Worstcase Control-relevant Identification," Automatica, Vol. 31, No. 12, 1995.

[7] P. A. Parrilo, R. S. Sánchez Peña and M. Sznaier, "A Parametric Extension of Mixed Time/Frequency Robust Identification," accepted for publication in the IEEE Trans. Automat. Contr., 1997.

[8] P. A. Parrilo, M. Sznaier, and R. S. Sánchez Peña, "Mixed time/frequency Robust Identification," Proceedings of the 35th Conference on Decision \& Control, Kobe, Japan, 1996. Also submitted to Automatica.

[9] R. S. Sánchez Peña and M. Sznaier, "Robust Identification with Mixed Time/Frequency Experiments: Consistency and Interpolation Algorithms," Proceedings of the 34th Conference on Decision $\&$ Control, New Orleans, USA, 1995.

[10] L. Vandenberghe and S. Boyd, "Semidefinite Programming," SIAM Review, Vol. 38, pp. 49-95, 1996.

[11] T. Zhou and H. Kimura, "Time Domain Identification for Robust Control," System \& Control Letters, Vol. 20, pp. 167-178, 1993. 